\title{
Was steckt hinter der Atemnot?
}

— Interessante Fälle aus der Praxis wurden beim 51. Pneumologenkongress in Hannover vorgestellt. Die erste Kasuistik, berichtet von Prof. Hans-Georg Predel aus Köln, macht besonders deutlich, wie eng pulmonale und kardiale Symptome zusammenhängen. Ein Feuerwehrmann klagte nach einem Einsatz über Husten und Atemnot. Zunächst wird angenommen, dass es sich um eine Reizung durch Rauchexposition handelt. Röntgen-Thorax und Spirometrie ergaben keinen pathologischen Befund. Nach der Gabe von Antitussiva und eines inhalativen Steroids (Beclometason) ließ der Reizhusten nach, aber die Belastungsdyspnoe blieb. Näheres Nachfragen ergab, dass die Dyspnoe schon länger bestanden hatte.

Bei einer anschließenden kardiologischen Abklärung stellte man eine Ejektionsfraktion von $45 \%$ fest (Vorbefund 60\%). Ruhe-EKG und Ruhespirometrie waren unauffällig. Bei der Ergospirometrie traten ventrikuläre Extrasystolen auf, die Herzfrequenz und der Blutdruck stiegen übermäßig an. Die $\mathrm{VO}_{2}$ war deutlich reduziert. Es fand sich kein Hinweis auf eine Kardiomyopathie oder Klap- penerkrankung. Auch eine Virusmyokarditis konnte serologisch ausgeschlossen werden. Positiv war jedoch die Serologie für Borrelien-Antikörper. Der Patient hatte eine Borreliose mit kardialer Beteiligung. Unter einer Therapie mit Doxycyclin und bei körperlicher Schonung erholte er sich vollständig.

\section{Erster Verdacht: Asthma bronchiale}

Einen weiteren Fall stellte Prof. Roland Buhl aus Mainz vor. Eine 31-jährigen Frau wurde mit einer obstruktiven Ventilationsstörung und progredienter respiratorischer Insuffizienz auffällig. Allergien sind aus der Vorgeschichte nicht bekannt. Die Patientin gab an, seit zwei Jahren nicht mehr zu rauchen. Der Hausarzt hatte wegen der eingeschränkten Lungenfunktion auf ein Asthma bronchiale getippt und eine Therapie mit einem inhalativen Steroid plus lang wirksamem Betamimetikum begonnen (als BeclometasonFormoterol-Fixkombination z.B. als Inuvair ${ }^{\circledR}$ erhältlich). Dies brachte aber keinen Erfolg. Bei der Untersuchung waren EKG und Echokardiografie normal. Auch eine neuromuskuläre Erkrankung wurde ausgeschlossen.

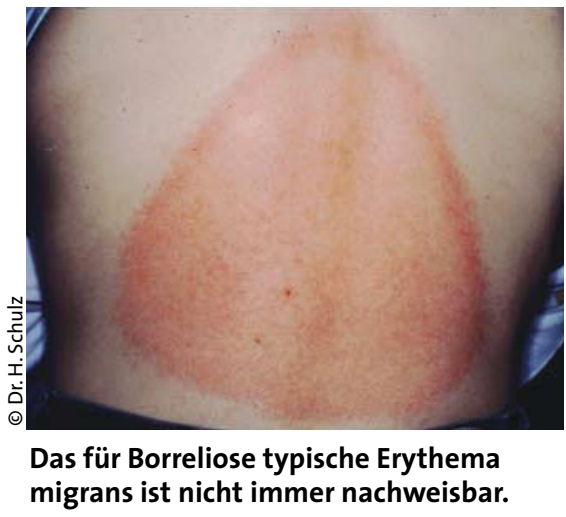

Fündig wurde man bei der Ergometrie unter Blutgasanalyse, die eine Methämoglobinämie von $18,5 \%$ (physiologisch < 1\%) bei einem regelrechten Sauerstoffpartialdruck erbrachte. Eine eingehende Befragung konnte klären, dass es sich um eine toxische Form handelte, hervorgerufen durch Poppers. Die Inhalation von Amyl- oder Butylnitrit führt dabei im Gehirn zu einer transienten Vasodilatation mit halluzinogenen Effekten. Unter Verzicht auf die Drogen besserte sich die Lungenfunktion in kurzer Zeit.

ab

Symposium „Der Pneumologe als Entscheider“, Pneumologenkongress, 18.3.2010 in Hannover, Veranstalter: Janssen-Cilag

Verschiedene Behandlungskonzepte bei pulmonalarterieller Hypertonie

\section{Hauptsache ist, frühzeitig einzugreifen}

— Die aktuelle europäische Leitlinie zur Therapie bei pulmonalerarterieller Hypertonie $(\mathrm{PAH})$ ist im Vergleich zu älteren Leitlinien ein wesentlicher Fortschritt, so Prof. Marius Hoeper, Hannover. Zu den wesentlichen Neuerungen zählen eindeutige Therapieempfehlungen für Patienten der NYHA-Klasse II, die nun mit Phosphodiesterase-5-Inhibitoren wie Sildenafil (Revatio ${ }^{\circledR}$ ) oder Endothelin-Rezeptorantagonisten behandelt werden sollen. Dies gilt auch für Deutschland. Eine eigene Leitlinie für die Bundesrepublik wird nicht erstellt, sondern nur eine deutsche kommentierte Fassung der ERC-ERS-Guidelines.

Das frühe therapeutische Eingreifen ist wichtig, so Hoeper, denn auch im frühen Stadium liegen bei den Patienten schon strukturelle Veränderungen in der Lungenstrombahn vor. Mit Ausnahme von Kindern, die sehr vasoreagibel sind, ist die Senkung des pulmonalarteriellen Drucks vermutlich nicht der primäre Wirkmechanismus der eingesetzten Medikamente. Deutlicher zeigt sich in Studien der Effekt auf die pulmonalvaskuläre Resistance.

\section{Erfolgskontrolle über die Gehstrecke} Der Erfolg der Therapie bei Erwachsenen darf nicht nur durch die Druckmessung kontrolliert werden, sondern durch Ziele wie das Erreichen der NYHA-Klasse II und einer 6-Minuten-Gehstrecke von $500 \mathrm{~m}$. Prof. Ardeschir Ghofrani, Bad Nauheim, stellte als mögliches Therapiekonzept eine stadienadaptierte Balance zwischen einfacher Anwendung („Convenience“) einerseits und Nebenwirkungsprofil andererseits vor. Im Frühstadium stehen Kalziumkanalblocker (bei positiven Va- soreagibilitätstest) im Vordergrund, $a b$ NYHA-Klasse II werden Phosphodiesterasehemmer, Endothelin-Rezeptorantagonisten oder inhalative Prostaglandinderivate eingesetzt. Im Stadium IV sind dann Therapien mit höheren Einschränkungen und Nebenwirkungen (intravenöse Gabe von Prostaglandinen) indiziert.

Alternativ bietet sich das Konzept „Hit hard and early“ an: Ähnlich wie in der Onkologie kommen hier im Frühstadium intensive Behandlungsschemata zum Einsatz, um einen möglichst großen Effekt zu erzielen. Im Spätstadium werden dann weniger belastende („palliative“) Therapien eingesetzt.

afi

„Pulmonale Hypertonie Up2Date“, München, 6.3.2010. Veranstalter: Pfizer 